\title{
Spatial attention in encoding letter combinations.
}

\author{
Mahalakshmi Ramamurthy ${ }^{1 *}$, Alex L. White ${ }^{2}$ Clementine Chou ${ }^{1}$, Jason D. Yeatman ${ }^{1}$ \\ ${ }^{1}$ Developmental-behavioral Pediatrics, School of Medicine \& Graduate school of Education, Stanford \\ University, CA, USA. \\ ${ }^{2}$ Department of Neuroscience E Behavior, Barnard College, NY, USA. \\ *Correspondence to: maha10@stanford.edu
}

\begin{abstract}
:
Reading requires the correct identification of letters and letter positions within words. Selective attention is, therefore, required to select chunks of the text for sequential processing. Despite the extensive literature on visual attention, the well known effects of spatial cues in simple perceptual tasks cannot inform us about the role of attention in a task as complex as reading. Here, we systematically manipulate spatial attention in a multi-letter processing task to understand the effects of spatial cues on letter encoding in typical adults. Overall, endogenous (voluntary) cue benefits were larger than exogenous (involuntary). We show that cue benefits are greater in the left than in the right visual field; and larger for the most crowded letter positions. Endogenous valid cues reduced errors due to confusing letter positions more than misidentifications, specifically for the most crowded letter positions. Therefore, shifting endogenous attention along a line of text is likely an important mechanism to alleviate the effects of crowding on letters within words. Our results help set the premise for building theories for how specific mechanisms of attention support reading development in children. Understanding the link between reading development and attention mechanisms has far reaching implications in the remediation for children with reading disabilities.
\end{abstract}

\section{Introduction}

Reading is a complex visual task that demands shifting and focusing spatial attention to extract information about the letters that compose words. As you read these lines, you are moving your eyes from word to word quite effortlessly, even though your visual field is cluttered with hundreds of letters. Selecting letters that form the current word and prioritizing the next word in the sentence are necessary steps to read fluidly. Indeed, due to a bottleneck in the word recognition circuitry ${ }^{1}$, multiple words compete with each other and focal attention is required to select one word at a time ${ }^{2,3}$. The ability to identify letters and extract their relative positions within each word is also fundamental for word recognition ${ }^{4}$. This is particularly true for children learning to read. While it is intuitive that selective attention is necessary for reading, the existing literature has yet to demonstrate how various mechanisms of attention affect the way letter combinations are processed. In this study we asked, how do two types of covert visual-spatial attention affect recognition of letters within a string? 
In the study of visual perception, spatial attention is defined as a collection of processes that prioritize task-relevant locations in the visual field and filter out irrelevant information. Spatial attention can be deployed overtly, by moving our eyes to a specific location, or covertly, without eye movements. Covert spatial attention helps monitor the environment and to plan subsequent eye movements. Covert spatial attention (henceforth simply referred to as "attention") can be directed to a certain location in space voluntarily, based on current goals, or involuntarily, due to sudden onset of a peripheral stimulus. The former type is endogenous attention, and the latter is exogenous. It has been shown that these types of attention improve task performance in a variety of visual tasks including contrast sensitivity, ${ }^{5,6}$ texture segmentation ${ }^{7}$, visual search ${ }^{8}$, and many others (for review see ${ }^{9}$ ). Despite the vast literature, it is difficult to extend our understanding of the effects of attention on processing low-level stimuli (e.g., Gabor patches) to highly specialized and complex visual tasks like reading.

Both endogenous and exogenous cues have been shown to improve performance for targets appearing at cued locations relative to targets at uncued locations, for many different stimuli and tasks ${ }^{10-14}$. However, the specific perceptual effects of endogenous and exogenous attention differ in key ways: i) the time course of exogenous and endogenous cueing effects are reported to differ ${ }^{15,16}$; ii) the magnitude of endogenous, but not exogenous, effects scale with the likelihood that relevant stimuli appear at the cued locations; iii) endogenous attention flexibly optimizes performance as a function of task demands (e.g: ${ }^{17-20}$; whereas exogenous cues cannot be ignored even when known to be uninformative ${ }^{21,22,23,21,22}$ and iv) there are qualitatively different effects reported with exogenous and endogenous cues. For example, in a texture segmentation task, exogenous attention improves performance at the periphery (where spatial resolution is low) but impairs performance at the center (where resolution is already high), whereas endogenous attention always improves perception by flexibly adjusting to spatial resolution ${ }^{20,24,25}$.

These differential effects limit us from extending and generalizing the effects of exogenous and endogenous attention from one context to another, especially, if the core question is in understanding the basic role of selection mechanisms involved in processing letter combinations. Therefore, we developed a task relevant to reading that allows for each type of covert spatial attention to be manipulated.

Word recognition is limited by the ability to identify its component letters ${ }^{4}$. To test the ability to identify letters within a string, we used the multi-element processing task ${ }^{26}$. First introduced by Sperling ${ }^{27}$, whole and partial report versions of the multi-element processing tasks are measures of information extraction from brief visual displays. In the whole report version, the task is to report the identity of as many letters as possible from a briefly displayed string. In the partial report, the task is to report one of the letters at a location that is cued after the stimulus disappears. 
In our task, the elements are letters (all consonants) that do not form real words. This ensures that accurate recognition must be done for each letter independently. This task is commonly cited in dyslexia literature. Some studies have reported that individuals with a sub-type of dyslexia exhibit deficits in both whole and partial report versions of the task ${ }^{28-30}$, and that this deficit might be causally related to reading development ${ }^{28,31}$. The reason for such a deficit is still not fully understood, some authors (e.g. ${ }^{28}$ ) attribute deficits in the multi-letter processing task to the "visual attention span", but the specific mechanism of attention that may or may not be involved has yet to be specified. Nevertheless, the multi-letter processing task reliably correlates with reading ability indicating the relevance of this measure for reading ${ }^{29,32}$. Here we systematically manipulate endogenous and exogenous spatial cues in the multi element processing task to specifically characterize the effects of attention on task performance.

\section{Materials and Methods}

Participants: We recruited 32 healthy adult participants ( 24 females, 8 males) between 18 and 35 years of age. Sample size was targeted to be twice that is typically targeted in visual attention studies that involve typical adult participants. All participants had normal or corrected-to-normal vision and gave written informed consent in accordance with the Stanford University Institutional Review Board. In addition, since the data collection was done during the pandemic, every measure was taken to comply with relevant guidelines at the time and to ensure safety of participants and experimenters. All experimental protocols were approved by the Stanford University Institutional Review Board. All participants had no known history of dyslexia, auditory deficits or attention deficits. Each participant also completed a battery of standard reading assessments (TOWRE test Torgesen, J., et al. 1999). Participants had a TOWRE composite score at or above the population average score of [range: 100 to 129; 50th to 97th percentile] and TOWRE Phonemic Decoding Efficiency score [range 100 to 132, 50th to 98th percentile]. 


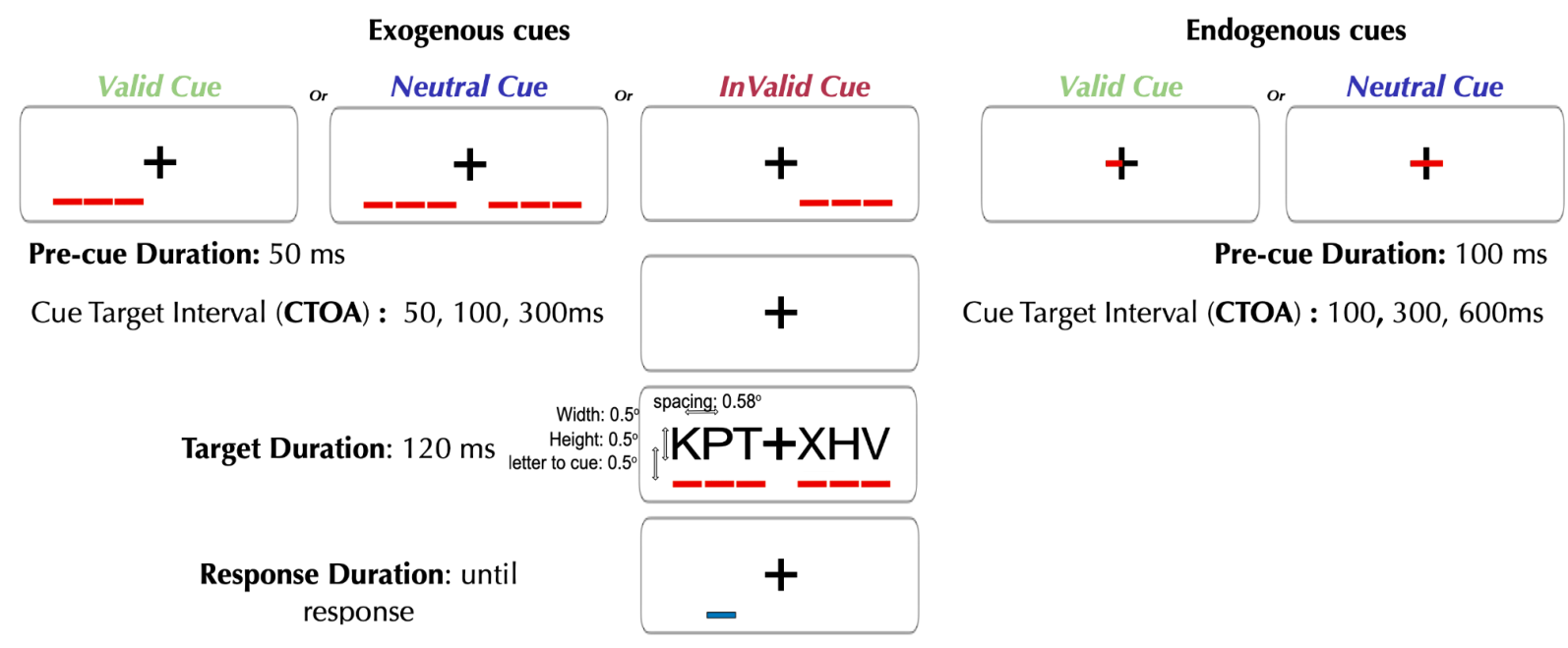

Task: Participants report the letter in the post-cued location from a set of 12 letters.

Fig. 1: Example trial sequence for the exogenous and endogenous attention experiments in the letter recognition task. On each trial the participant is presented with a briefly flashed string of 6 letters, three to the left and three to the right of fixation. Each letter spanned $0.5^{\circ}$ of visual angle with $0.58^{\circ}$ center-to-center spacing at $1.16 \mathrm{x}$ the letter width. Immediately after the letters disappear, a "post-cue" appears: a blue line under one of the letter locations. The participant's task is to report the identity of the target letter that had been at the post-cue location. They do so by clicking on one of 12 letters presented below the post-cue (not shown). Spatial attention is manipulated before the letters appear by "pre-cues" that direct attention to either the left three letters or to the right three letters. Exogenous pre-cues are three red lines that flash briefly below the letter locations. They are uninformative, meaning that their locations are random with respect to the post-cued target's location. Endogenous pre-cues are short red line segments next to the central fixation mark that point to the left side, right side or both. They are informative, meaning that they indicate the side where the target will be. Therefore, endogenous pre-cues manipulate the voluntary deployment of attention towards task-relevant information, while exogenous pre-cues manipulate the involuntary capture of attention by salient stimuli. In all cases, participants are required to maintain central fixation (monitored by an eye tracker). For both types of cues, there is a "neutral" condition in which both sides are pre-cued, and the target could be at either side. A "valid" pre-cue is one that is on the same side as the upcoming post-cue. An "invalid" pre-cue is one that is on the opposite side of the post-cue. The effect of attention is measured via "cue effects": the difference in target recognition accuracy between valid, neutral, and invalid trials. Note that the pre-cues are represented with the target strings in the figure to show the spacing between the letters and the pre-cue. Pre-cues appear before the target string in every trial sequence. 
Equipment and Stimulus: We generated stimuli using MATLAB (The Mathworks Corporation, Natick, MA, USA) and the Psychophysics Toolbox ${ }^{33,34}$ on Linux (Ubuntu 16.04). We used the ViewPixx EEG display (1920x1080 resolution, $120 \mathrm{~Hz}$ refresh rate) that subtended 36 degrees of visual angle $\left(36^{\circ}\right)$ horizontally. Participants were comfortably seated at a head and chinrest 73 $\mathrm{cm}$ from the monitor, and used the mouse to select their response from the screen. The screen background was set to white $\left(100 \mathrm{~cd} / \mathrm{m}^{2}\right)$ and a black $\left(4.3 \mathrm{~cd} / \mathrm{m}^{2}\right)$ fixation cross $\left(0.5^{\circ} \times 0.5^{\circ}\right)$ was always present at the center of the screen. The target stimuli were a string of six letters, three to the left of the fixation cross and three to the right of the fixation cross. Six consonants were randomly sampled without replacement from a set of 12 letters $[\mathrm{B}, \mathrm{F}, \mathrm{H}, \mathrm{K}, \mathrm{L}, \mathrm{N}, \mathrm{P}, \mathrm{T}, \mathrm{V}, \mathrm{X}, \mathrm{Y}, \mathrm{Z}]$, that match on perimetric properties ${ }^{35}$, for each trial. All letters were in monospaced Dejavu font at $100 \%$ contrast. Letter height was set to $0.5^{\circ}$ and the center-to-center letter spacing was $0.58^{\circ}$. Each six letter string subtended $3.98^{\circ}$.

Trial sequence: An illustration of trial sequence with an exogenous and endogenous spatial cue is presented in Fig. 1. On each trial, after the pre-cue (described below), the participant is presented with a string of 6 letters flashed for $120 \mathrm{~ms}$, three to the left and three to the right of fixation. Immediately after the letters disappear, a "post-cue" appears: a blue line under one of the letter locations. The participant's task is to report the identity of the target letter that was at the post-cue location, by clicking on one letter from a set of 12 letter choices provided. The 12 letter choices comprise of all the 6 letters (including 1 target) presented in the string along with 6 letters that were not part of that trial's string. Spatial attention was manipulated before the letters appear by "pre-cues" that direct attention to either the left three letters or to the right three letters.

Pre-cues: To elicit exogenous shifts of spatial attention to either the left or right side, the cues were red lines flashed for $50 \mathrm{~ms}$ under the three letter positions before the onset of the string. The exogenous cues were uninformative as to the side of the target letter to be post-cued. To elicit endogenous shifts of spatial attention to the left or right side, the cue was a salient red line presented adjacent to the center on one of the horizontal arms of the fixation cross, indicating with $100 \%$ certainty the side of the target letter to be post-cued. Participants were explicitly instructed about the uninformative and informative nature of the cue in the former and latter conditions respectively. The exogenous and endogenous cue conditions were run on separate days. The exogenous cue condition was always run before the endogenous condition.

In all cases, participants were required to maintain central fixation (see eye tracking methods below). For both types of cues, there was a "neutral" condition in which both sides are pre-cued, and the target could be at either side. A "valid" pre-cue is one that is on the same side as the upcoming post-cue. An "invalid" pre-cue (which only applies for exogenous) appears on the opposite side of the post-cue. In the exogenous cue condition, there is equal probability of a 
cue being valid, invalid or neutral, whereas half the endogenous cues were neutral and the rest were all valid.

Cue-target onset asynchrony (CTOA): The time interval between the onset of a pre-cue and onset of the stimulus string is the cue-target onset asynchrony abbreviated as CTOA. We used CTOAs of 50, 100 and $300 \mathrm{~ms}$ for the exogenous cues and CTOAs of 100, 300 and $600 \mathrm{~ms}$ for the endogenous cues. These choices allowed us to measure the dynamics of the two cueing effects within their appropriate range. The reflexive, transient, exogenous system is known to operate at shorter intervals than the sustained, voluntary, endogenous system ${ }^{15,16,36}$.

There were 10 trials for each position (6 positions), for each of the CTOAs (3 CTOAs) and for each cue validity condition ( 3 for exogenous and 2 for endogenous cue conditions) totalling to 540 trials in the exogenous and 360 trials in the endogenous cue condition.

Eye tracking: Eye position was monitored using the Eyelink 1000 plus (SR research, Ontario, Canada). Any horizontal saccades greater than the $0.5^{\circ}$ during the presentation of the letter string aborted the trial, which was added back to the end of the block. Any fixation outside of the screen center $+/-0.5^{\circ}$ horizontally and $2^{\circ}$ vertically pauses the experiment for a recalibration. Observers made fixation breaks on less than $5 \%$ of trials. Less than $1 \%$ of the eye gaze positions, across all participants, were outside of the central fixation window ( $1 \mathrm{deg}$ ) during the period from the onset of the cue to the offset of the target for both the exogenous and endogenous cue conditions. Thus results are not due to eye movements towards the cued sides.

Attentional effects: The effect of attention is measured as the difference in target recognition accuracy between valid, neutral and invalid trials. Based on extensive research on characterizing exogenous and endogenous attention ${ }^{9}$ we expect task performance with valid cues to be greater than that with neutral cues (and invalid, in the case of exogenous cue). The cue benefit is the difference in target recognition sensitivity between valid and neutral cue trials. For exogenous pre-cues, the cue cost is the cost of directing attention to the wrong side: the difference in sensitivity between neutral and invalid cue trials. Finally, the total cue effect, for the exogenous system, is the difference in sensitivity between valid and invalid cues.

Inclusion criteria: All participants had normal or corrected normal vision, with no known history of dyslexia or attention disorder. Not all participants were native English speakers but all standardized reading scores were average or above average (range: 100 - 132).

Data Analysis: Of the 32 subjects recruited, 4 were excluded from all the analysis: one who had $\mathrm{ADHD}$, and three who could not return for the second visit. We used linear mixed effects model (LME) analyses and post-hoc t-tests to analyze accuracy across different CTOAs, letter positions, 
and cue validity conditions. The random effects consist of subject-dependent random intercept and slopes. In LME fixed and random effects explain differences between subjects and the variability within subjects respectively. We used a maximal random effects structure (random intercepts and slopes ${ }^{37}$ ), in all our LME models unless specified.

Reliability: For each participant, for each cue validity condition, we computed d' separately on odd and even trials The correlation, across subjects, between those split-half $d^{\prime}$ levels indicates reliability. A Spearman-Brown correction was applied to the obtained correlation to adjust for the fact that only half the trials were used. The Spearman-Brown split-half reliabilities for d' in the exogenous and endogenous neutral cue conditions were $r=0.91$ and 0.89 , respectively. For valid trials, reliabilities for the exogenous and endogenous cues were $r=0.91$ and $r=0.87$, respectively.

\section{$\underline{\text { RESULTS }}$}

\section{Exogenous and endogenous spatial cues affect sensitivity in a multi-letter processing task.}

We first established that exogenous and endogenous cues affect accuracy for identifying a single post-cued letter when participants are briefly presented with a horizontal string of 6 random letters centered on the fovea. Fig. 2 shows accuracy $\left(d^{\prime}\right)$ averaged across all participants $(n=28)$ for each cue validity condition and CTOA for both exogenous (Fig. 2a) and endogenous (Fig. 2b) cue types. In general, the partial report task was difficult and accuracy was consistently below 80\% correct [Exogenous (M+/-SEM \%): 73.03+/-1.98; Endogenous: 76.60+/-2.02]. Overall mean performance $\left(d^{\prime}\right)$ in the neutral cue condition was 2.33 (SE: 0.07). Notably, performance in the neutral cue trials (blue bars in Fig. 2) remained consistent across different CTOAs for both cue types [effect of CTOA for exogenous: $\mathrm{F}(2,81)=0.9876$; $\mathrm{p}=0.38$, and endogenous $\mathrm{F}(2,81)=0.5209$; $\mathrm{p}=0.59]$, indicating that dividing attention is not affected by the interval between the cue and the target.

To examine the effect of exogenous spatial cues on task performance we fit a linear mixed effects (LME) model to the accuracy data $\left(d^{\prime}\right)$ with fixed effects of cue validity and CTOA, and a maximal random effects structure. $d^{\prime}$ in the valid condition was greater than in the neutral condition, which was greater than the invalid condition. Valid exogenous cues (when the pre-cued side matched the post-cued side) improved performance relative to neutral exogenous cues by, on average, $5.41 \%$ accuracy ( $d^{\prime}$ difference of $\left.0.22 ; t(247)=5.35, p=1.94 \times 10^{-07}\right]$. Invalid exogenous cues (when the pre-cued side was opposite to that of the post-cued side) decreased performance compared to neutral cues, on average, by $9.38 \%$ accuracy ( $\mathrm{d}^{\prime}$ difference of 0.32 ; $\left.t(247)=-7.93, p=7.76 \times 10^{-14}\right]$. Sensitivity also varied across CTOAs $[F(2,247)=5.92, p=0.0031]$. Posthoc $\mathrm{t}$-tests revealed that the CTOA of $50 \mathrm{~ms}$ was significantly different from $100 \mathrm{~ms}[\mathrm{t}(247)=$ 2.21, $\mathrm{p}=0.028]$ and $300 \mathrm{~ms}[\mathrm{t}(247)=3.38, \mathrm{p}=0.00084]$. 
In the endogenous condition, valid cues increased performance by, on average, $6.98 \%$ accuracy $\left(d^{\prime}\right.$ difference 0.31 ) compared to neutral cues $\left[F(1,164)=69.18 ; p=3.29 \times 10^{-14}\right]$. Sensitivity also varied across CTOAs $\left[\mathrm{F}(2,164)=15.25 ; \mathrm{p}=8.44 \times 10^{-7}\right]$; post hoc t-tests showed that all three CTOAs were different from each other [100:300ms $t(164)=-5.15 ; p=7.19 \times 10^{-7} \& 100: 600 \mathrm{~ms}$ $\left.\mathrm{t}(128)=-8.31 ; \mathrm{p}=3.29 \times 10^{-14}\right]$, with higher $\mathrm{d}^{\prime}$ with valid cues at the longest CTOA.
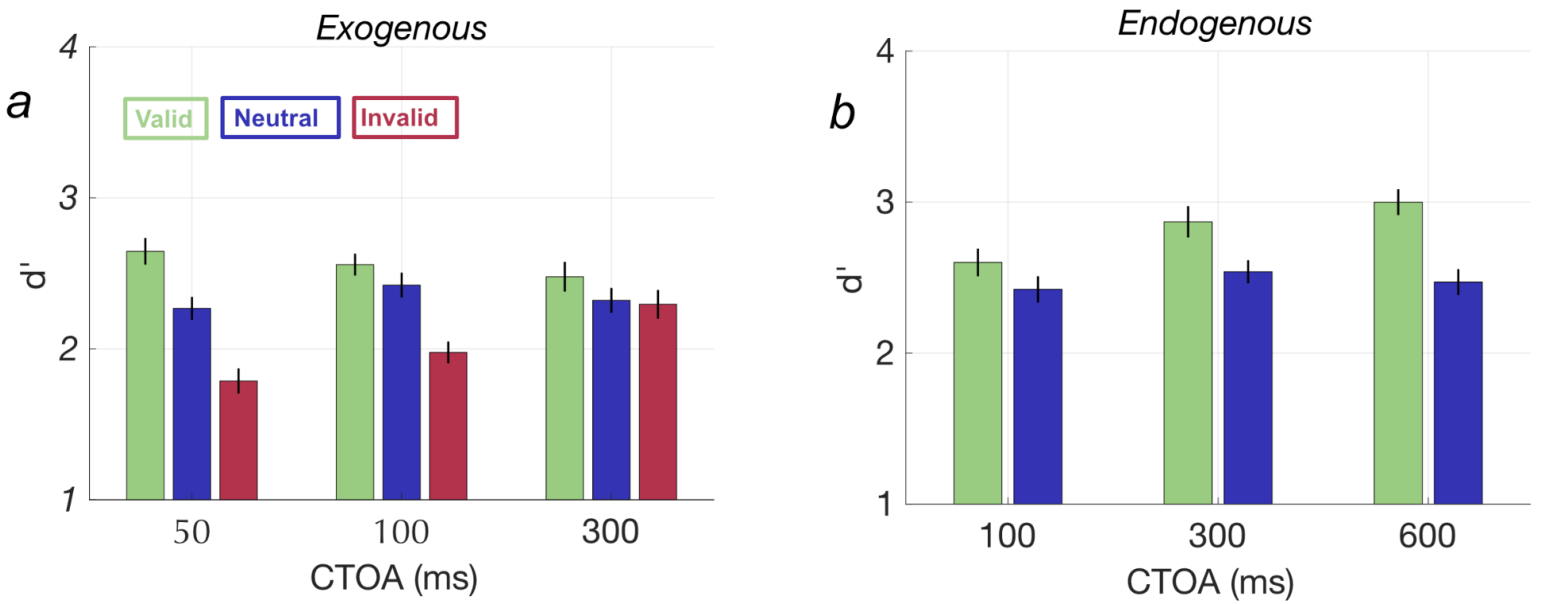

Fig. 2. shows accuracy in letter recognition in the multi-letter processing task as a function of cue-target asynchrony. (a) results from the exogenous cue type and (b) results from endogenous cue type, across different cue validity trials (colors) and CTOAs. Error bars represent SEM.

\section{Exogenous and endogenous attention exhibit different temporal dynamics.}

Based on prior studies, we expect the exogenous attention system to respond more quickly (within $\sim 100 \mathrm{~ms}$ ) to cues compared to the endogenous deployment of attention ( $\sim 300$ to $500 \mathrm{~ms})^{9}$. The interval between the onset of the cue and the onset of the target is typically considered to be the interval during which attentional resources are allocated to the cued side ${ }^{38}$. To evaluate how cue benefits (valid - neutral d') change over time, we fit a LME model to cue benefits with CTOAs as fixed effects for each cue type. Fig. 3 shows cue benefits for both exogenous (Fig. 3a) and endogenous cues (Fig. 3b) as a function of CTOA. Exogenous cue benefits were greatest at the shortest time interval and decayed at longer intervals $\left[\mathrm{F}(2,81)=14.1, \mathrm{p}=5.57 \times 10^{-06}\right]$; post hoc t-tests: $50 \mathrm{~ms}>100 \mathrm{~ms}: \mathrm{t}(81)=3.99 ; \mathrm{p}=1.04 \times 10^{-04}$ and $\left.100>300 \mathrm{~ms}: \mathrm{t}(81)=2.95 ; \mathrm{p}=0.004\right]$. Endogenous cue benefits, on the other hand, increased as a function of CTOA, with maximum effect at the longest interval $\left[\mathrm{F}(2,81)=8.88 ; \mathrm{p}=3.24 \times 10^{-04}\right.$; post hoc t-tests: $600 \mathrm{~ms}>100 \mathrm{~ms}$ : $\mathrm{t}(81)=4.11, \mathrm{p}=6.46 \times 10^{-05}$ and $\left.300 \mathrm{~ms}>100 \mathrm{~ms}: \mathrm{t}(81)=2.31, \mathrm{p}=0.023\right]$.

Like the exogenous cue benefit, the exogenous cue cost (neutral - invalid) also has a time course (also see Supplementary). Fig. 3a shows exogenous cue benefit, cue cost and total cue effect as a function of CTOA. A LME model was fit to cue cost with fixed effects of CTOA. Cue cost significantly varied as a function of CTOA $\left[\mathrm{F}(2,81)=17.61 ; \mathrm{p}=4.46 \times 10^{-07}\right]$, being greatest at $50 \mathrm{~ms}$, and declining thereafter $\left[300<50 \mathrm{~ms}: \mathrm{t}(81)=5.55 ; \mathrm{p}=3.48 \times 10^{-7} ;\right.$ post hoc t-tests: $300<100$ 
ms: $\left.\mathrm{t}(81)=5.23, \mathrm{p}=1.27 \times 10^{-06}\right]$. The cue cost at a CTOA of $100 \mathrm{~ms}$ was not significantly different from that at $50 \mathrm{~ms}(\mathrm{t}(81)=-0.52 ; \mathrm{p}=0.60)$. Both exogenous benefits and costs were lower at a CTOA of $300 \mathrm{~ms}$, suggesting that the effects decay with time for exogenous attention.

The following sections focus on the time points when each attention system has its maximal effects: $50 \mathrm{~ms}$ for exogenous cues and $600 \mathrm{~ms}$ for endogenous cues. Note that the exogenous cue benefits at $600 \mathrm{~ms}$ were slightly higher than exogenous cue benefits at $50 \mathrm{~ms}$ (Mean d' difference=: 0.151; SE: 0.07; $\mathrm{t}(27)=2.12 ; \mathrm{p}=0.041$ ).
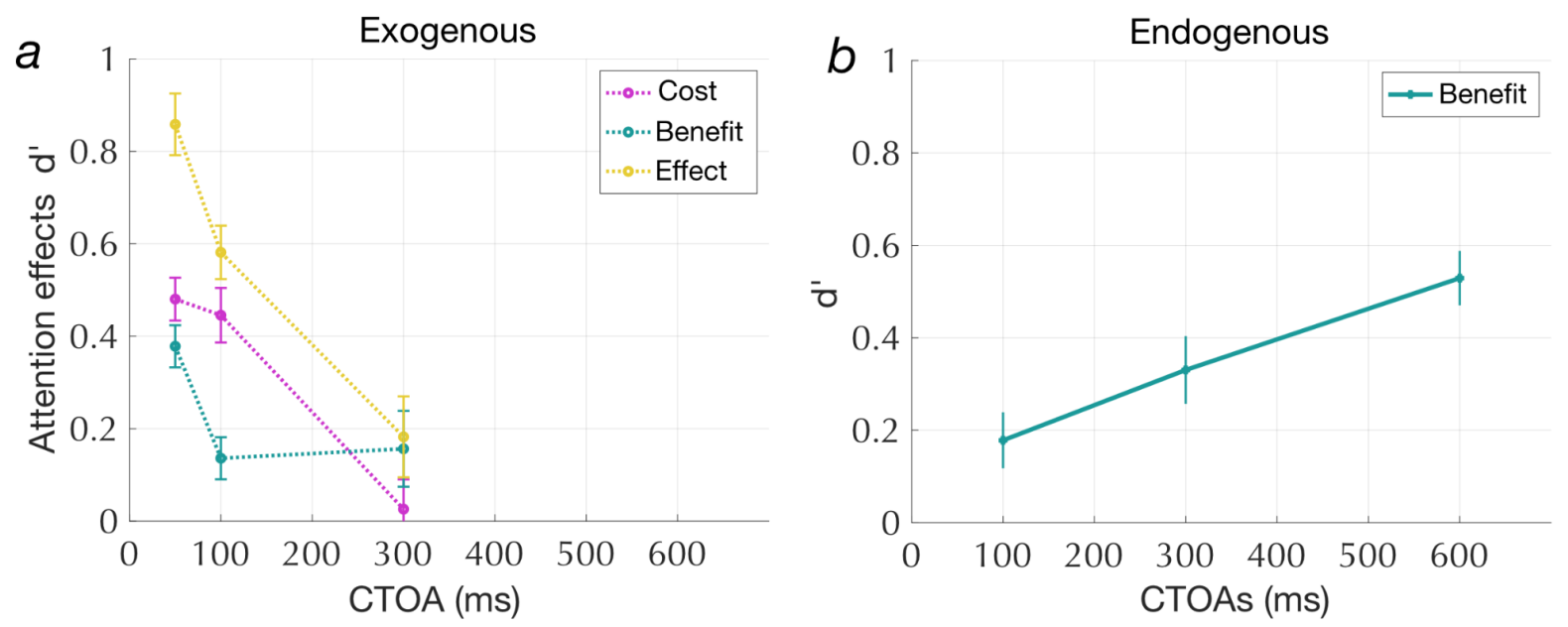

Fig. 3. (a) Time trace of cue benefits, cue cost and cue effects with exogenous cues CTOAs: 50, 100, 300ms); (b) Endogenous cue benefits as a function of CTOAs (100, 300, 600ms). Error bars represent SEM.

\section{Cue benefits interact with letter position within a string.}

Many studies have shown that letter recognition accuracy differs across positions within a string $35,39,40$. In general, accuracy as a function of position follows a W-shape, with greater accuracy for outer positions and at fovea, and diminished accuracy for internal letters ${ }^{35,40}$. Consistent with the literature, we also see a $\mathrm{W}$-shaped serial position function for letters in all conditions and cue types (see Fig. $4 \mathrm{a}$ and b for exogenous cues at $50 \mathrm{~ms}$ and endogenous cues at 600ms, and Supplementary Fig. S1 and S2 show the overlapping CTOAs). The W-profile for letter recognition is thought to be due to a combination of various underlying mechanisms ranging from the drop in acuity with visual eccentricity, visual crowding and working memory 35,39. Interestingly, we noticed that letter recognition was significantly better in the right compared to the left hemifield, replicating well-known hemifield asymmetries for word (and letter recognition) (Mishkin and Gorgays 1952; Bouma 1973; Nazir et al. 1991; Legge et al. 2001). The asymmetry was present for both exogenous and endogenous cues [Neutral cue condition: significant effect of cue type $F(1,332)=6.87 ; p=0.009$ and hemifield $\left.F(1,322)=16.01 ; p=7.74 \times 10^{-5}\right]$.

Here we ask a novel question: does the serial position function interact with spatial attention? Specifically, do the effects of exogenous and endogenous pre-cues differ across letter 
positions in a string? To examine these questions, we first plot exogenous and endogenous cue benefits (valid-neutral) as a function of the post-cued letter position: we call this the cue benefit function (again computed with CTOAs 50 and $600 \mathrm{~ms}$, respectively). If a valid cue simply increases letter recognition accuracy equally across all letter positions, then we would expect the cue benefit function to be flat. On the one hand, if attention further enhances letters in positions that already show high sensitivity (i.e., positions 1, 4, and 6 which have high sensitivity) then the cue benefit function would also be W-shaped. If, on the other hand, letters in crowded positions (i.e., in the troughs of the $\mathrm{W}$-profiles) benefit more with an attentional cue, then the cue benefit function would be M-shaped.

Figures $4 \mathrm{c}$ and $4 \mathrm{~d}$ show the cue benefit functions. We fit a LME model to cue benefits with both cue type and positions as fixed effects. We observed a significant main effect of position $\left[\mathrm{F}(5,324)=11.27 ; \mathrm{p}=4.94 \times 10^{-10}\right]$ and cue type $[\mathrm{F}(1,324)=6.40, \mathrm{p}=0.01]$ but no significant interaction between cue type and positions $[\mathrm{F}(5,324)=1.49 ; \mathrm{p}=0.19]$. A post-hoc t-test (with a Bonferroni adjusted $p$-threshold for multiple comparisons set to: $\mathrm{p}_{\text {Adjusted }}=0.010$ ) comparing benefits at each position revealed that cue benefits only at position 1 was significantly lower than the average cue benefits across all other positions $\left[\mathrm{t}_{1}(324)=-3.42 ; \mathrm{p}_{1}=7.1 \times 10^{-4}\right]$. Cue benefits at positions 2 and 3 were significantly higher than the average across all other positions $\left[\mathrm{t}_{2}(324)=4.04, \mathrm{p}_{2}=6.53 \times 10-5 ; \mathrm{t}_{3}(324)=3.17, \mathrm{p}_{3}=0.002\right]$. Thus the $\mathrm{M}$-shaped cue benefit function shows that the serial position function interacts with spatial cues and exogenous and endogenous attention both enhance sensitivity to crowded letter positions. Next, we asked how cue benefits vary between hemifields. Specifically, how does attention interact with the right hemifield bias for encoding letter strings?

\section{Letters are easier to recognize in the right visual field, but cue benefits are larger in the left.}

We observe a significant hemifield asymmetry with both cue types (see inset plots in Fig. 4c , 4d and Fig. 5a). Fitting a LME on cue benefits with cue type and positions as fixed effects showed that cue benefits were significantly larger for the letter positions in the left hemifield (positions: 1,2 , and 3) compared to the right hemifield (positions: 4, 5 and 6) $[F(1,332)=10.17 ; p=0.0016]$. As previously reported, cue benefits were larger overall for endogenous than exogenous cues $[\mathrm{F}(1$, $332)=5.41 ; \mathrm{p}=0.021]$. However the interaction between cue type and hemifield was not significant $[F(1,332)=0.87 ; p=0.35]$. Thus, spatial attention effects are greater in the left hemifield, where letter recognition accuracy is lower. 

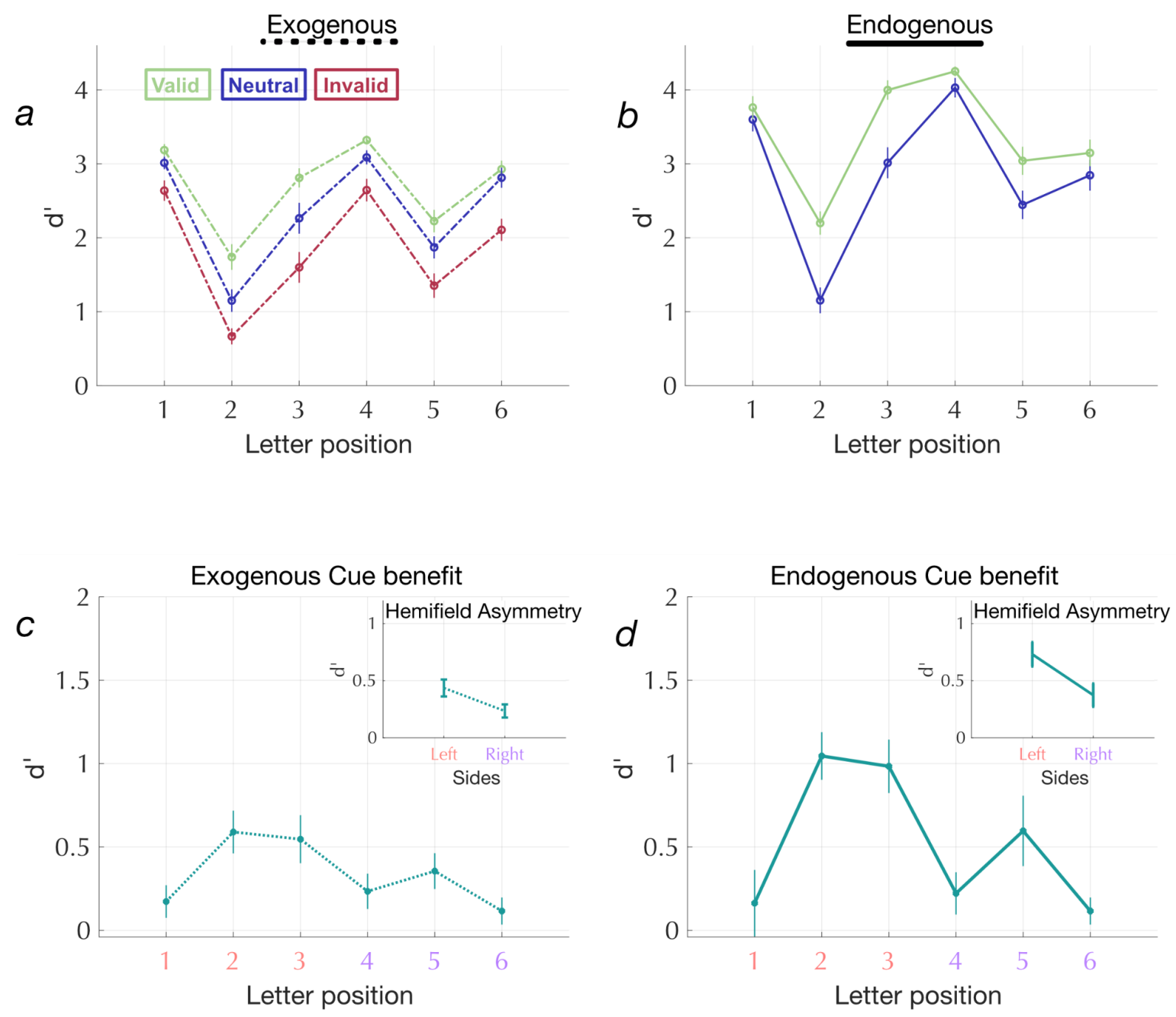

Fig. 4: $(\boldsymbol{a}, \boldsymbol{b})$ Serial position functions are W-shaped for both cue types. (c, d) cue-benefit functions. Both exogenous and endogenous cue benefits show $\mathrm{M}$-shaped profiles with larger cueing effects at crowded letter positions.

Cue benefits are larger for letters in the most crowded positions within the string.

A letter that can be easily identified when presented alone becomes unrecognizable when presented alongside nearby flankers, a phenomenon called visual crowding ${ }^{41-43}$. To compare cue benefits at the most crowded and least crowded positions, we took the outermost letters at positions 1 and 6 and compared them to the crowded letter positions 2 and 5. Figure 5b shows that cue benefits for the crowded letter positions ( 2 and 5 ) were higher than the outermost letters (1 and 6). A LME model is fit to cue benefits with cue type (exogenous or endogenous) and letter crowding (crowded or uncrowded) as fixed effects. There was significant main effects of letter crowding $\left[\mathrm{F}(1,220)=15.88 ; \mathrm{p}=9.165 \times 10^{-5}\right]$, but not of cue type $[\mathrm{F}(1,220)=2.83 ; \mathrm{p}=0.09]$ nor 
a significant interaction $[F(1,220)=1.45 ; p=0.23]$ (Fig. 5b). Thus, both exogenous and endogenous cues benefit those letters in the crowded positions more than those in the uncrowded positions.
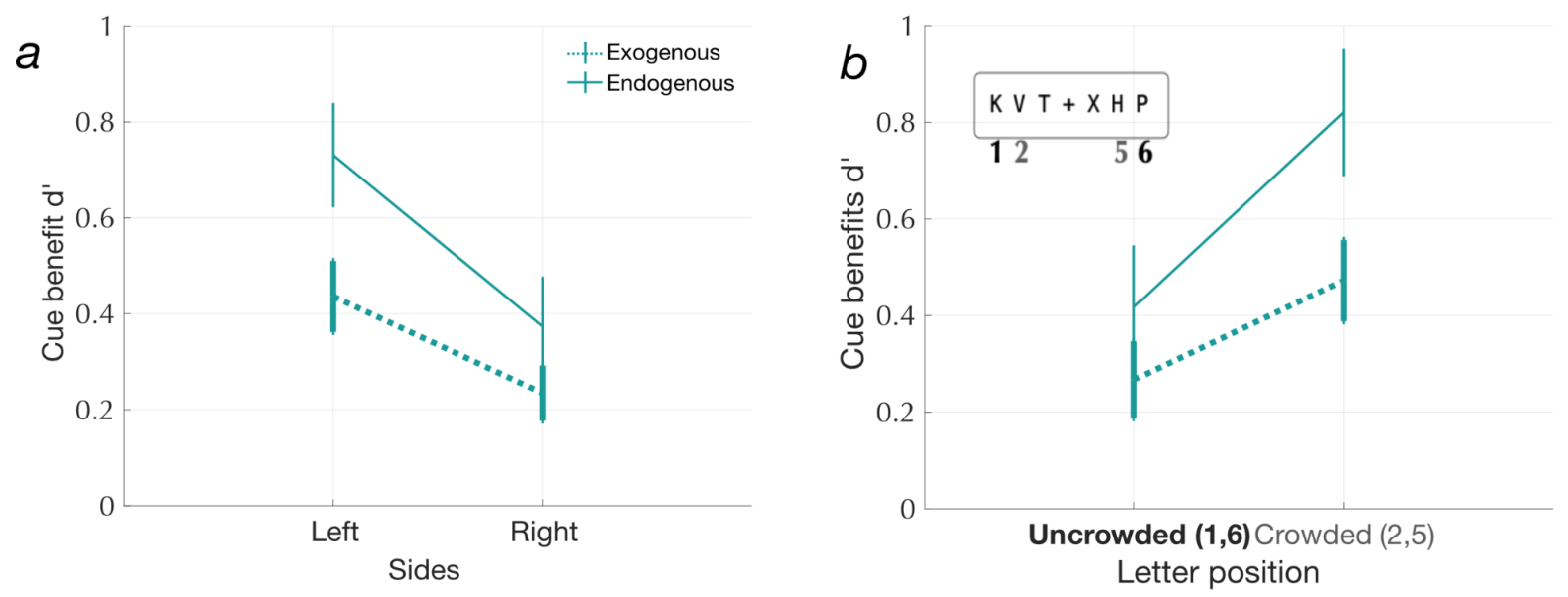

Fig. 5. (a) cue benefits for the left and right hemifield. We see a significant main effect of cue type and hemifield but no significant interaction effect. $(b)$ cue benefit for the most crowded and the least crowded letter positions. We see a significant main effect of letter crowding but no significant effect of cue type and no interaction between cue type and letter crowding.

\section{How do spatial cues reduce errors?}

To understand how exogenous and endogenous cues increase letter recognition accuracy within a string, we evaluated the types of errors made on incorrect trials. A transposition error is when the participant confuses the relative positions and reports an adjacent letter in the string instead of the target letter. For example, in a target string "K N B T Y P", reporting T or P would be classified as a transposition error if the post-cued target was $\mathrm{Y}$. A misidentification error is when the participant reports a letter that was not present in the string. For example, in the same target string, a misidentification error would be reporting $\mathrm{V}$ instead of the target $\mathrm{Y}$.

We computed the proportions of both error types as the number of errors divided by the total number of trials, separately for each position and cue condition. The resulting error profile is shown in Figures 6a and 6b. Note that the error profile is the inverse of the $\mathrm{W}$-shaped profile for accuracy. Error rates are higher (at each position) with neutral cues (blue curves) than with valid cues (green curves). With invalid exogenous cues (red curves), errors are higher compared to neutral cues. This difference in error rates with a valid compared to a neutral cue is expressed as the difference in proportion errors between the two conditions $(\mathrm{N}-\mathrm{V})$.

We then assessed the difference in these error rates between valid and neutral cue conditions to better understand how valid cues improve overall accuracy. Since differences in proportions are non-linear, we first linearize the proportion error data by putting them through the inverse of the cumulative normal distribution function. We then defined $\Delta$ error as the 
difference between those normalized error rates in the neutral - valid conditions (see norminv for calculations, Fig. $6 \mathrm{c}$ and d).

We then fit a LME model to $\triangle$ error for each cue type with letter position and error type as fixed effects. With exogenous cues, $\Delta$ errors showed a significant effect of letter position $\left[\mathrm{F}(5,324)=4.71 ; \mathrm{p}=3.6 \times 10^{-4}\right]$ but no significant effect of error type $[\mathrm{F}(1,324)=0.12 ; \mathrm{p}=0.73]$ or interaction between error type and letter position $[\mathrm{F}(5,324)=0.44 ; \mathrm{p}=0.82]$. A post hoc $\mathrm{t}$-test (with a Bonferroni adjusted $\mathrm{p}$-threshold for multiple comparisons: $\mathrm{p}_{\text {Adjusted }}=0.010$ ) showed that $\Delta$ errors at position 1 were significantly lower compared to the average $\Delta$ errors across all other positions $\left(\mathrm{t}_{1}(324)=-2.81 ; \mathrm{p}=0.005\right)$. It should be noted that $\Delta$ errors at the first letter position are close to zero because valid cues don't benefit recognition much at position 1 . With endogenous cues, $\Delta$ errors showed a significant effect of letter position $\left[F(5,324)=7.89 ; \mathrm{p}=4.94 \times 10^{-7}\right]$, no significant effect of error type $[\mathrm{F}(1,324)=2.72 ; \mathrm{p}=0.09]$, but a significant interaction between letter position and error type $[\mathrm{F}(5,324)=2.87 ; \mathrm{p}=0.015]$. A post hoc t-test showed that transposition errors at position 2 were significantly higher compared to errors at all other positions $\left(t_{2}(324)=\right.$ 2.55; $\mathrm{p}=0.01$ ). Thus a larger cue benefit in the left hemifield with both endogenous and exogenous valid cues (shown in Figures $4 \mathrm{c}$ and $4 \mathrm{~d}$ ) is due to a reduction in both transposition and misidentification errors. Interestingly, only with endogenous valid cues we observe a significant interaction between error type and letter position showing that valid endogenous cues reduce transposition errors for the most crowded letter position in the hemifield with reduced sensitivity (position 2 ). 


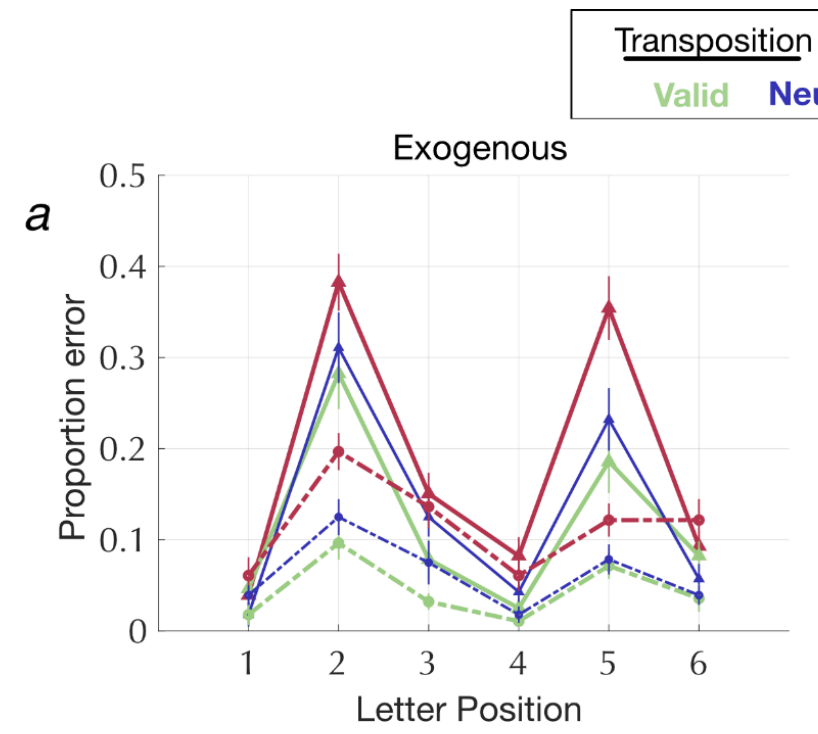

Neutral Invalid

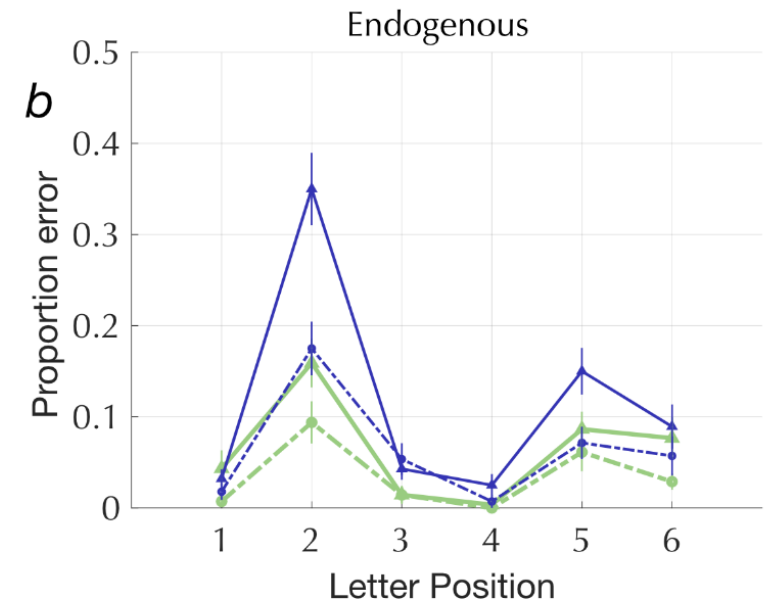

Transposition Misidentification
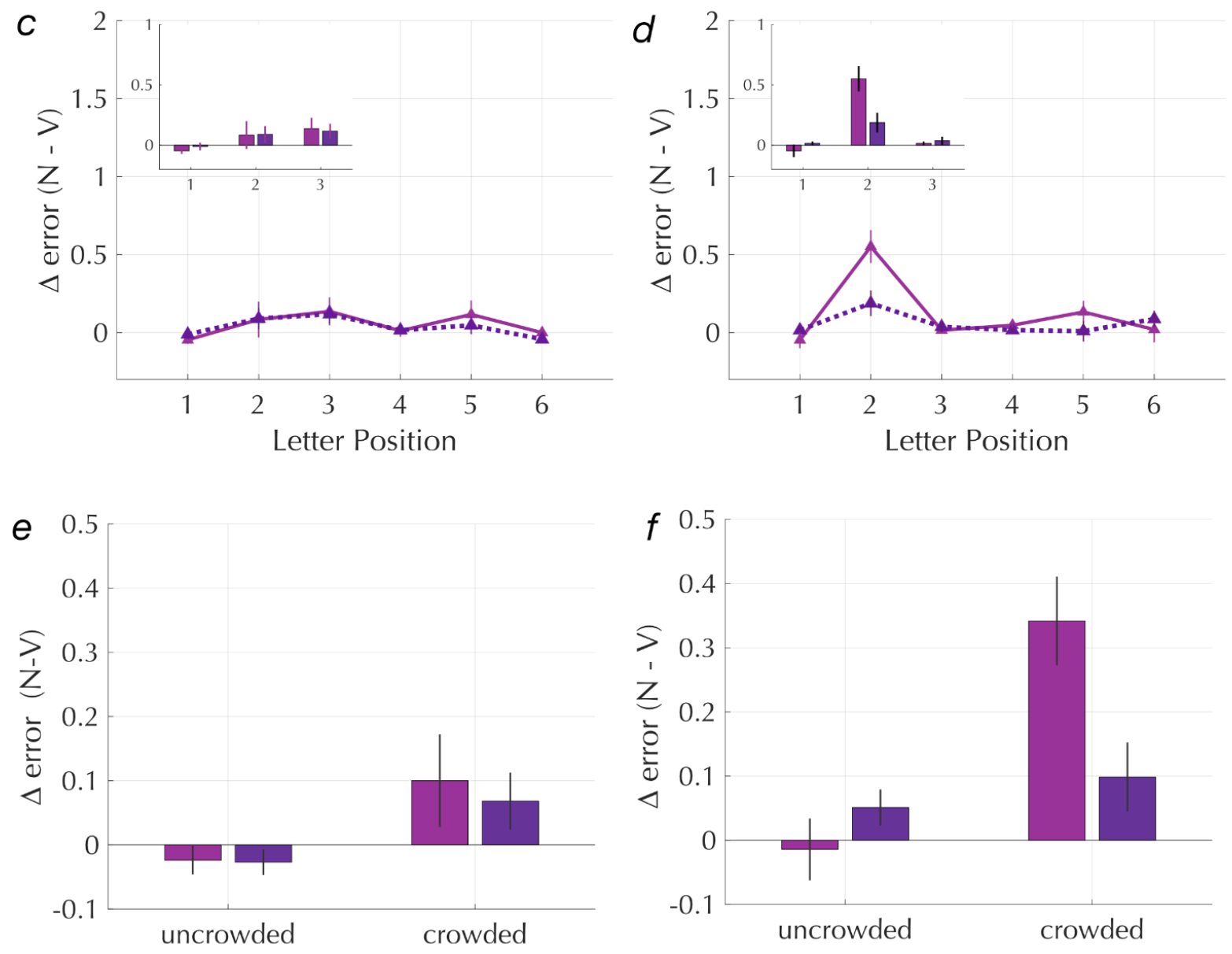
Fig. 6: (left: Exogenous and right: endogenous). a and b: shows error profiles, proportion transposition and misidentification errors as a function of letter position for both trial types with neutral cues (blue lines), valid cues (green lines) and invalid cues (red lines). Transposition errors are $\sim 2 x$ more likely than misidentification errors overall. $\mathbf{c}$ and $\mathbf{d}$ : shows the linearized difference in errors with a neutral vs. valid cue. Exogenous valid cues reduce transposition errors uniformly across all letter positions, however endogenous valid cues decrease both transposition and misidentification errors in the crowded positions more compared to uncrowded positions. The inset figures show the same information as bar graphs for the three letter positions in the left hemifield. It is clear that endogenous valid cues reduce transposition errors for the most crowded position in the left hemifield. e and $\mathbf{f}$ : Errors calculated for letter positions 1 and 6 (uncrowded) and positions 2 and 5 (crowded). We show that endogenous valid cues reduce transposition errors particularly for those letters in the crowded positions.

To understand how the two cue benefits interact with letter crowding (shown in Fig. 5b), we analyzed how the cues affect the proportion of misidentification and transposition errors for crowded positions ( 2 and 5 ) and uncrowded positions (1 and 6). A three way mixed effects model was fit to the $\Delta$ errors with cue type (exogenous or endogenous); error type (transposition or misidentification) and letter crowding (crowded or uncrowded) as fixed effects and a full random structure. There was a significant main effect of letter crowding $[F(1,440)=20.56$; $\mathrm{p}=0.00001]$, with greater $\Delta$ error for the crowded than uncrowded positions; a significant main effect of cue type $[F(1,440)=6.875 ; \mathrm{p}=0.009]$, with larger reductions in errors with valid endogenous than exogenous cues; and a significant three way interaction between letter crowding, error type and cue type $[\mathrm{F}(1,440)=4.148 ; \mathrm{p}=0.038]$ showing that the interaction between letter crowding and error type $[F(1,440)=6.034 ; p=0.012]$ varies with the type of cue (see Fig. 6e and f). A post hoc comparison showed that transposition errors were significantly reduced in the crowded letter positions with endogenous valid cues $[t(98.35)=4.223$; bonferroni adjusted $\mathrm{p}$ value for multiple comparison $\mathrm{p}=0.0015]$. Thus, although both exogenous and endogenous cues benefit the crowded letter positions more than the uncrowded letter positions, endogenous valid cues reduce transposition errors to a greater extent than exogenous valid cues for crowded letters (seen in Fig. 6f).

\section{Discussion}

We compared the effects of exogenous (reflexive, involuntary) and endogenous (sustained, voluntary) spatial attention on performance in the multi-letter processing task. Our results show that both exogenous and endogenous attention affect letter recognition, increasing accuracy at the cued location and decreasing accuracy at the uncued locations. Consistent with previous

studies $9,22,44$, we observe that exogenous and endogenous attention operate at different time scales (Fig. 3). Exogenous cue benefits were greatest at the shortest cue-to-target interval (50 ms) 
and then declined, whereas endogenous cue benefits were greatest at the longest cue-to-target interval (600ms).

For both cue types, valid cues improved accuracy the most for those letter positions where accuracy was the lowest in the neutral cue condition. Specifically, cue benefits were higher for the left hemifield and for the middle (positions 2 and 5) letter positions. Endogenous cue benefits were generally larger than exogenous cue benefits. Valid endogenous cues were especially effective at reducing transposition errors for the most crowded letters in the left visual field. In other words, our results suggest that endogenous attention (volitional control of attentional resources) helps in correctly encoding letter order within a string, a skill fundamental to word recognition.

Many authors purport the idea that visual attention plays a causal role in dyslexia (the most prevalent learning disability). However many studies do not isolate the role of specific attentional mechanisms, and many do not clearly operationalize "attention" 28-31,45-47. Others who have operationalized attention show correlations with cueing effects but with tasks that have no clear relevance to reading ${ }^{46-49}$ ). Some such paradigms that don't clearly isolate exogenous and endogenous attentional mechanisms $\left({ }^{49}\right)$. Our data are the first to compare the effects of endogenous and exogenous spatial cues, in terms of letter identity and letter order perception and time course.

The processing of text - specifically letters within a word (string) - is affected by many inherent limitations of the visual system that results in the $\mathrm{W}$-shaped position function for letter

recognition within a string ${ }^{35,39,50-52}$. For example, internal letters are more difficult to identify due to crowding $\left({ }^{35,39,50-52}\right)$, but there are other mechanisms at play as well $\left({ }^{35,39,50-52} 7\right)$. Whatever the underlying mechanisms are that result in the $\mathrm{W}$-shaped profile, those factors can be ameliorated by voluntary focusing of spatial attention which ultimately helps encode letter order. It is interesting that we found these effects even though the spatial cues were not directed to specific letter positions, but rather to just one side (3 letters on the left or three on the right). Therefore, voluntarily focusing attention to a subset of letters helps in recognizing their order correctly.

Endogenous attention is likely to be particularly important for the process of learning to read because it enhances the encoding of information at letter positions where sensitivity is the lowest. It is a feature of the endogenous attentional system to flexibly adapt to task specific demands, as has been reported in other tasks like texture segmentation ${ }^{20,53}$.

\section{Conclusion}

Our results are the first to isolate the role of different spatial attentional mechanisms in letter encoding, a process fundamental to word recognition. We have systematically characterized the effect of two distinct selection processes in a task relevant for reading, and our results demonstrate some similar and some distinct effects on how readers encode the identity and 
position of letters within a string. This work lays the foundation for two important future lines of research: i) evaluating specific attention mechanisms in reading disability, based on concrete hypothese. For example, based on our findings we can hypothesize that endogenous attention is critical for reading, particularly in young children learning to read; and ii) tracking the developmental trajectory of attentional mechanisms to further understand how development shapes attention and intersects with the development of reading ability.

Data Availability:

Data supporting this work is available from the project's git repository [https://github.com/yeatmanlab/SpatialAttention LetterEncoding.git].

\section{Code Availability:}

Code to reproduce figures and analyses is available from the project's git repository [https://github.com/yeatmanlab/SpatialAttention_LetterEncoding.git].

\section{Acknowledgements:}

This work was supported by research funding from Microsoft, NICHD R01HD09586101 and Jacobs Research Fellowship to J.D.Y and Stanford Maternal and Child Health Research Institute through the Postdoctoral Fellowship Awarded to M. R.

\section{Author contribution:}

Mahalakshmi Ramamurthy developed the study concept. Mahalakshmi Ramamurthy and Alex L. White and Jason D. Yeatman contributed to the study design. Testing and data collection were performed by Mahalakshmi Ramamurthy and Clementine Chou. Mahalakshmi Ramamurthy performed the data analysis and interpretation under the supervision of Alex L. White and Jason D. Yeatman. Mahalakshmi Ramamurthy drafted the manuscript, and Alex L. White and Jason D. Yeatman provided critical revisions. All authors reviewed the manuscript and approved the final version for submission.

\section{Conflicts of Interests:}

The authors declare no conflicts of interest with respect to the authorship or the publication of this article.

\section{References:}

1. White, A. L., Palmer, J., Boynton, G. M. \& Yeatman, J. D. Parallel spatial channels converge at a bottleneck in anterior word-selective cortex. Proc. Natl. Acad. Sci. U. S. A. 116, 10087-10096 (2019). 
2. White, A. L., Palmer, J. \& Boynton, G. M. Visual word recognition: Evidence for a serial bottleneck in lexical access. Atten. Percept. Psychophys. 82, 2000-2017 (2020).

3. White, A. L., Palmer, J. \& Boynton, G. M. Evidence of Serial Processing in Visual Word Recognition. Psychol. Sci. 29, 1062-1071 (2018).

4. Pelli, D. G., Farell, B. \& Moore, D. C. The remarkable inefficiency of word recognition. Nature 423, 752-756 (2003).

5. Dosher, B. A. \& Lu, Z. L. Perceptual learning reflects external noise filtering and internal noise reduction through channel reweighting. Proc. Natl. Acad. Sci. U. S. A. 95, 13988-13993 (1998).

6. Carrasco, M. \& McElree, B. Covert attention accelerates the rate of visual information processing. Proc. Natl. Acad. Sci. U. S. A. 98, 5363-5367 (2001).

7. Yeshurun, Y. \& Carrasco, M. Attention improves or impairs visual performance by enhancing spatial resolution. Nature 396, 72-75 (1998).

8. Nakayama, K. \& Mackeben, M. Sustained and transient components of focal visual attention. Vision Res. 29, 1631-1647 (1989).

9. Carrasco, M. Visual attention: The past 25 years. Vision Res. 51, 1484-1525 (2011).

10. Cave, K. R. \& Bichot, N. P. Visuospatial attention: beyond a spotlight model. Psychon. Bull. Rev. 6, 204-223 (1999).

11. Anllo-Vento, L. Shifting attention in visual space: the effects of peripheral cueing on brain cortical potentials. Int. J. Neurosci. 80, 353-370 (1995).

12. Ruz, M. \& Lupiáñez, J. A review of attentional capture: On its automaticity and sensitivity to endogenous control. Psicológica (2002).

13. Di Russo, F., Martínez, A. \& Hillyard, S. A. Source analysis of event-related cortical activity during visuo-spatial attention. Cereb. Cortex 13, 486-499 (2003).

14. Eimer, M. \& Kiss, M. Involuntary attentional capture is determined by task set: evidence from event-related brain potentials. J. Cogn. Neurosci. 20, 1423-1433 (2008).

15. Klein, R. M. On the Control of Visual Orienting. in Cognitive neuroscience of attention , ( $p p$ (ed. Posner, M. I.) vol. 466 29-44 (The Guilford Press, xiii, 2004).

16. Funes, M. J., Lupiáñez, J. \& Milliken, B. Separate mechanisms recruited by exogenous and endogenous spatial cues: evidence from a spatial Stroop paradigm. J. Exp. Psychol. Hum. Percept. Perform. 33, 348-362 (2007).

17. Hein, E., Rolke, B. \& Ulrich, R. Visual attention and temporal discrimination: Differential effects of automatic and voluntary cueing. Visual Cognition vol. 13 29-50 (2006).

18. Yeshurun, Y., Montagna, B. \& Carrasco, M. On the flexibility of sustained attention and its effects on a texture segmentation task. Vision Res. 48, 80-95 (2008).

19. Barbot, A., Landy, M. S. \& Carrasco, M. Differential effects of exogenous and endogenous attention on second-order texture contrast sensitivity. Journal of Vision vol. 12 6-6 (2012).

20. Barbot, A. \& Carrasco, M. Attention Modifies Spatial Resolution According to Task Demands. Psychol. Sci. 28, 285-296 (2017).

21. Yantis, S. \& Jonides, J. Abrupt visual onsets and selective attention: evidence from visual search. J. Exp. Psychol. Hum. Percept. Perform. 10, 601-621 (1984).

22. Müller, H. J. \& Rabbitt, P. M. A. Spatial Cueing and the Relation between the Accuracy of 'Where' and 'What' Decisions in Visual Search. The Quarterly Journal of Experimental 
Psychology Section A vol. 41 747-773 (1989).

23. Giordano, A. M., McElree, B. \& Carrasco, M. On the automaticity and flexibility of covert attention: A speed-accuracy trade-off analysis. Journal of Vision vol. 930-30 (2009).

24. Jigo, M. \& Carrasco, M. Endogenous and exogenous covert attention differentially modulate second-order textures. Journal of Vision vol. 181259 (2018).

25. Yeshurun, Y. \& Carrasco, M. The effects of transient attention on spatial resolution and the size of the attentional cue. Percept. Psychophys. 70, 104-113 (2008).

26. Averbach \& E. Short term storage of information in vision. Contemporary Theory and Research in Visual Perception 202-214 (1968).

27. Sperling, G. The information available in brief visual presentations. Psychological monographs: General and applied 74, 1 (1960).

28. Bosse, M.-L., Tainturier, M. J. \& Valdois, S. Developmental dyslexia: the visual attention span deficit hypothesis. Cognition 104, 198-230 (2007).

29. Lobier, M., Zoubrinetzky, R. \& Valdois, S. The visual attention span deficit in dyslexia is visual and not verbal. Cortex 48, 768-773 (2012).

30. Lallier, M., Donnadieu, S. \& Valdois, S. Developmental dyslexia: exploring how much phonological and visual attention span disorders are linked to simultaneous auditory processing deficits. Ann. Dyslexia 63, 97-116 (2013).

31. Prado, C., Dubois, M. \& Valdois, S. The eye movements of dyslexic children during reading and visual search: impact of the visual attention span. Vision Res. 47, 2521-2530 (2007).

32. Onochie-Quintanilla, E., Defior, S. \& Simpson, I. C. Visual multi-element processing as a pre-reading predictor of decoding skill. J. Mem. Lang. 94, 134-148 (2017).

33. Brainard, D. H. The Psychophysics Toolbox. Spat. Vis. 10, 433-436 (1997).

34. Pelli, D. G. The VideoToolbox software for visual psychophysics: transforming numbers into movies. Spat. Vis. 10, 437-442 (1997).

35. Castet, E., Descamps, M., Denis-Noël, A. \& Colé, P. Letter and symbol identification: No evidence for letter-specific crowding mechanisms. J. Vis. 17, 2 (2017).

36. Posner, M. I. \& Cohen, Y. Components of visual orienting. Attention and performance X: Control of language processes 32, 531-556 (1984).

37. Barr, D. J., Levy, R., Scheepers, C. \& Tily, H. J. Random effects structure for confirmatory hypothesis testing: Keep it maximal. J. Mem. Lang. 68, (2013).

38. Posner, M. I., Snyder, C. R. \& Davidson, B. J. Attention and the detection of signals. J. Exp. Psychol. 109, 160-174 (1980).

39. Tydgat, I. \& Grainger, J. Serial position effects in the identification of letters, digits, and symbols. J. Exp. Psychol. Hum. Percept. Perform. 35, 480-498 (2009).

40. Mason, M. Recognition time for letters and nonletters: effects of serial position, array size, and processing order. J. Exp. Psychol. Hum. Percept. Perform. 8, 724-738 (1982).

41. Pelli, D. G. Crowding: a cortical constraint on object recognition. Curr. Opin. Neurobiol. 18, 445-451 (2008).

42. Levi, D. M. Visual crowding. Curr. Biol. 21, R678-9 (2011).

43. Bouma, H. Visual interference in the parafoveal recognition of initial and final letters of words. Vision Res. 13, 767-782 (1973).

44. Liu, T., Stevens, S. T. \& Carrasco, M. Comparing the time course and efficacy of spatial and 
feature-based attention. Vision Res. 47, 108-113 (2007).

45. Hari, R. \& Renvall, H. Impaired processing of rapid stimulus sequences in dyslexia. Trends Cogn. Sci. 5, 525-532 (2001).

46. Franceschini, S., Gori, S., Ruffino, M., Pedrolli, K. \& Facoetti, A. A causal link between visual spatial attention and reading acquisition. Curr. Biol. 22, 814-819 (2012).

47. Facoetti, A., Ruffino, M., Peru, A., Paganoni, P. \& Chelazzi, L. Sluggish engagement and disengagement of non-spatial attention in dyslexic children. Cortex 44, 1221-1233 (2008).

48. Roach, N. W. \& Hogben, J. H. Attentional modulation of visual processing in adult dyslexia: a spatial-cuing deficit. Psychol. Sci. 15, 650-654 (2004).

49. White, A. L., Boynton, G. M. \& Yeatman, J. D. The link between reading ability and visual spatial attention across development. Cortex 121, 44-59 (2019).

50. Castet, É., Descamps, M., Denis-Noël, A. \& Colé, P. Dyslexia Research and the Partial Report Task: A First Step toward Acknowledging Iconic and Visual Short-term Memory. Sci. Stud. Read. 24, 159-169 (2020).

51. Scaltritti, M., Dufau, S. \& Grainger, J. Stimulus orientation and the first-letter advantage. Acta Psychol. 183, 37-42 (2018).

52. Reuther, J. \& Chakravarthi, R. Categorical membership modulates crowding: Evidence from characters. Journal of Vision vol. 145 (2014).

53. Jigo, M. \& Carrasco, M. Differential impact of exogenous and endogenous attention on the contrast sensitivity function across eccentricity. J. Vis. 20, 11 (2020). 